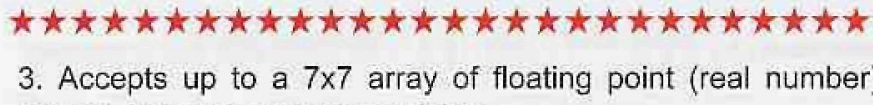
values, for much greater precision.

4. Can scale the results with a floating point number, or automatically for maximum unclipped contrast.

5. Reads Photoshop format files AND ALSO plain ascii text files.

6. Works in Photoshop and all compatible programs on both Mac and Windows computers.

Encouraged by the recent response by readers of the microscopy listserver to our offering of a free plug-in to draw magnification bars on micrographs, we are offering this plug-in for free download and use. It is one of nearly 200 plug-ins in the widely used Fovea Pro package, but can be used without installing or owning that package.

The plug-in, along with instructions and some example filter files, can be downloaded as a .sit file for Macintosh or a zip file for Windows from:

<http://www.reindeergraphics.com/free.html\#custom>.

While you are there, please take a look at the full range of Fovea Pro capabilities, which include comprehensive tools for image processing and analysis. Also, check out some of the other free downloads that are available.

John Russ, North Carolina State University

Dr JohnRuss@aol.com
A Home-made Antifade Medium

\section{for Fluorescent Dyes}

Most antibody companies (i.e., Dako, Vector, Molecular $\star$ Probes) sell a medium which contains an antifade medium. This $\star \star$ can also be easily prepared in one's own lab. Here is my favorite * formulation, which also contains DAPI (Blue fluorescent nuclear counterstain), but it can be left out.

$25 \mathrm{mg} / \mathrm{mL}$ 1,4-diazabicyclo[2.2.2]octane (DABCO) with $0.5 \mathrm{mg} / \mathrm{mL} 4,6$-diamidino-2-phenylindole (DAPI),

in $50 \%$ glycerol/phosphate buffered saline, $\mathrm{pH} 8.6$.

DABCO is dissolved in $100 \%$ glycerol then double-strength $\star$ phosphate-buffered saline at $\mathrm{pH} 8.6$ is added in equal volume to $\hbar$ the glycerol. The DAPI can be added from a higher concentra- $\star$ tion solution stock which can be stored frozen. All reagents can $\star$ be purchased from Sigma Chemical. $\mathrm{pH}$ is very important.

(Note: mention of a vendor does not constitute endorsement, 1 * just use these particular products.)

Tim Plummer, Mayo Clinic plummer.timothy@mayo.edu

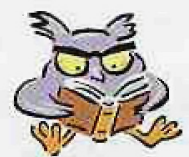

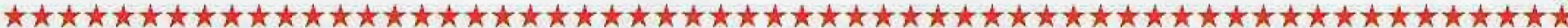

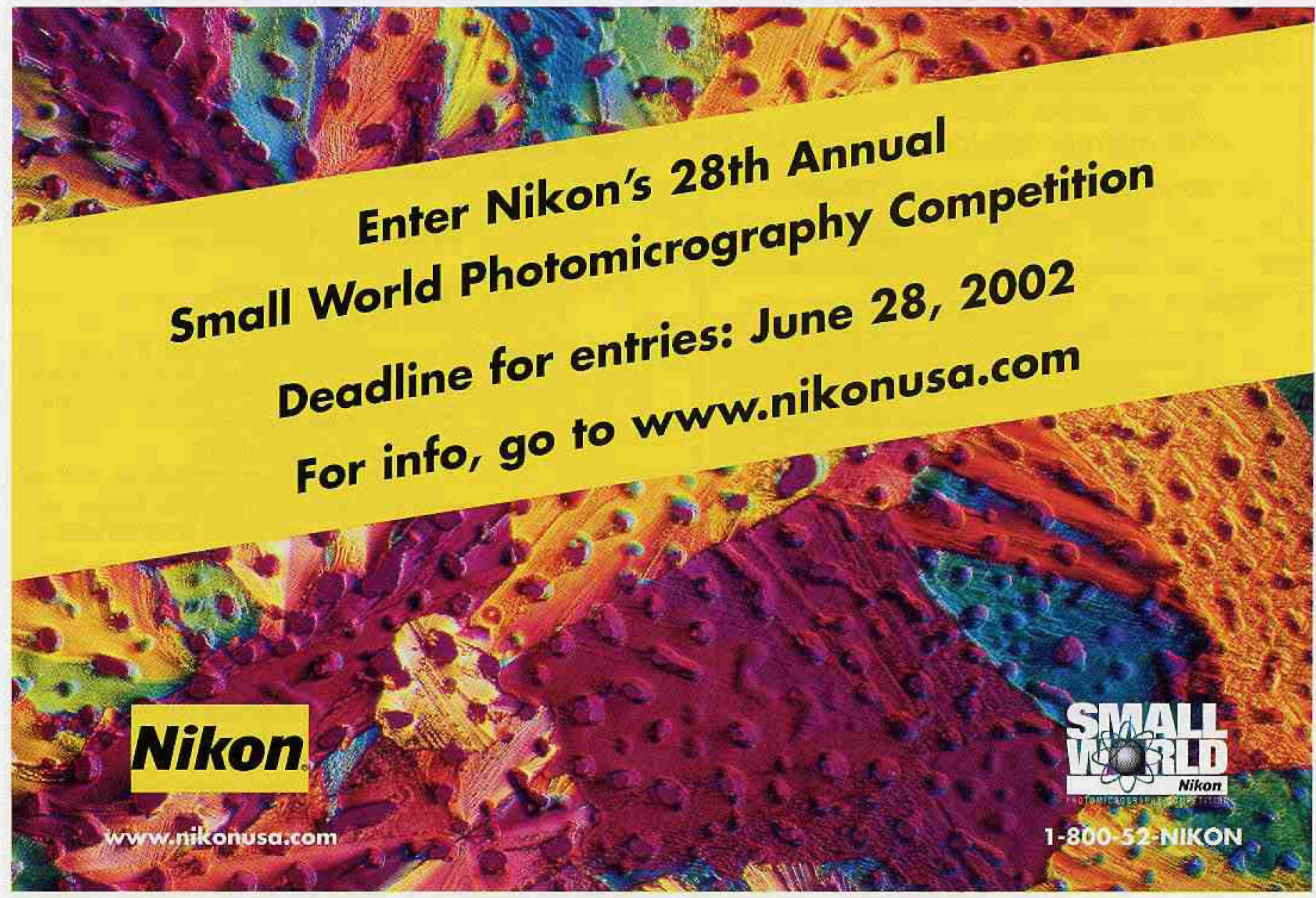

